\section{Selected Abbreviations Used in This Manual}

A adenine; adenosine

Ac acetyl

ACE acetoxyethoxy; acetoxyethyl

ADA adenosine deaminase

ADP adenosine 5'-diphosphate

ADTT 3-amino-1,2,4-dithiazoline-

5-thione

AEEA 2-aminoethoxy-2-ethoxy acetic

acid

AFM atomic force microscopy

AMP adenosine 5'-monophosphate

AMV-RT avian myoblastosis virus reverse transcriptase

ANA arabinonucleic acid

AP alkaline phosphatase

3-APA 3-aminopicolinic acid

APS ammonium persulfate

Ar aryl

ASY average stepwise yield

ATP adenosine 5'-triphosphate

ATPase adenosine triphosphatase

ATT 6-aza-2-thiothymine

AU absorbance units

AUFS absorbance units full scale

B base (nucleobase)

BAP bacterial alkaline phosphatase

BCIP 5-bromo-4-chloro-3-indolyl

phosphate

BDT 3H-1,2-benzodithiol-

3-one-1,1-dioxide

BHOC benzhydryloxycarbonyl

BIT benzimidazolium triflate

BMPM 1,1-bis(4-methoxyphenyl)-1-

pyrenylmethyl

Bn benzyl

bNA branched nucleic acid

BOC tert-butyloxycarbonyl

BOMP 2-(benzotriazol-1-yloxy)-

1,1-dimethyl-2-(pyrrolidin-1-yl)-1,3,2-

diazaphospholidinium

hexafluorophosphate

BSA bovine serum albumin;

bis(trimethylsilyl)acetamide

Bu butyl

Bz benzoyl

C cytidine; cytosine
cAMP adenosine $3^{\prime}, 5^{\prime}$-cyclic-

monophosphate

CD circular dichroism

CDI 1,1'-carbonyldiimidazole

cDNA complementary deoxyribonucleic

acid

CDP cytidine 5'-diphosphate

CE capillary electrophoresis; cyanoethyl

$\boldsymbol{C}^{\mathbf{e x}}$ excess heat capacity

CHA $\alpha$-cyano-4-hydroxycinnamic acid

Ci curie

CID collision-induced dissociation

CMCT 1-cyclohexyl-3-(2-

morpholinoethyl)carbodiimide

metho-p-toluene sulfonate

CMP cytidine 5'-monophosphate

COSY correlation spectroscopy

CPG controlled-pore glass

CPI cyclopropapyrroloindole

CPK Corey-Pauling-Koltun (molecular models)

cpm counts per minute

CPMB Current Protocols in Molecular

Biology

CSA D-10-camphorsulfonic acid

CSO (1S)-(+)(10-camphorsulfonyl)-

oxaziridine

CSP calf spleen phosphodiesterase

CTAB cetyltrimethylammonium

Ctmp 1-(2-chloro-4-methylphenyl)-4-

methoxypiperidin-4-yl

CTP cytidine 5'-triphosphate

dA deoxyadenosine

Dabcyl 4-[4-(dimethylamino)phenyl]-

azobenzoic acid

dADP deoxyadenosine diphosphate

dAMP deoxyadenosine monophosphate

DAP diaminopurine

DAST (diethylamino)sulfur trifluoride

dATP deoxyadenosine triphosphate

DBMB 2-(dibromomethyl)benzoyl

DBPNC 2,6-dibromo-4-benzoquinone-

$N$-chloroimine

DBU 1,8-diazabicyclo[3.4.0]undecene-

7-ene

dC deoxycytosine 
DCA dichloroacetic acid

DCC 1,3-dicyclohexylcarbodiimide

dCDP deoxycytosine diphosphate

DCHA dicyclohexylamine

DCI 4,5-dicyanoimidazole

DCM dichloromethane

dCMP deoxycytosine monophosphate

dCTP deoxycytosine triphosphate

DDQ 2,3-dichloro-5,6-

dicyano-1,4-benzoquinone

DEAD diethyl azodicarboxylate

DEC 1-(3-dimethylaminopropyl)-3-

ethylcarbodiimide

DEMA diethoxymethyl acetate

DEPC diethylpyrocarbonate

dG deoxyguanosine

DG distance geometry

dGDP deoxyguanosine diphosphate

dGMP deoxyguanosine monophosphate

dGTP deoxyguanosine triphosphate

DHB dihydroxybenzoic acid

DHPC dihexanoylphosphatidylcholine

dI deoxyinosine

DIAD diisopropyl azodicarboxylate

DIP 4,7-diphenyl-1,10-phenanthroline

DIPEA or DIEA diisopropylethylamine

DMAEDE 2'-O-2-[2-( $N, N-$

dimethylamino)ethyloxy]ethyl

DMAoE 2'-O-[2-(N,N-

dimethylaminooxy)ethyl

DMAP 4-dimethylaminopyridine

DMBOC 3',5'-dimethoxybenzoin-

oxycarbonyl

DMEM Dulbecco's minimal essential

medium (or Dulbecco's modified Eagle's

medium)

Dmf dimethylaminomethylene

DMF dimethylformamide

DMS dimethyl suberimidate; dimethyl

sulfate

DMSO dimethylsulfoxide

DMPC dimyristoylphosphatidylcholine

DMTr 4,4'-dimethoxytrityl

DNA deoxyribonucleic acid

DNBSB 2-(2,4-dinitrophenylsulfenyl-

oxymethyl)benzoyl

dNDP deoxynucleoside diphosphate

dNMP deoxynucleoside monophosphate

DNP 2,4-dinitrophenyl

DNPEOC 2-(2,4-dinitrophenyl)-

ethoxycarbonyl

dNTP deoxynucleoside triphosphate

DODC 3,3'-diethyloxadicarbocyanine

DPC diphenylcarbamoyl

Selected

DPI 1,2-dihydro-3H-pyrrolo[3,2-e]indole-

carboxylate

dpm disintegrations per minute
DSC differential scanning calorimetry;

disuccinimidyl carbonate

dsDNA double-stranded DNA

dT deoxythymidine

dTDP deoxythymidine diphosphate

DTE dithioerythritol

dTMP deoxythymidine monophosphate

DTMT 2-(isopropylthiomethoxymethyl)-

benzoyl

DTr 4-decyloxytrityl $\left(\mathrm{C}_{10} \mathrm{Tr}\right)$

DTT dithiothreitol

dTTP deoxythymidine triphosphate

dUTP deoxyuridine triphosphate

DVB divinylbenzene

DX double cross-over

EC Enzyme Commission

EDC 1-[3-(dimethylamino)propyl]-3-

ethylcarbodiimide

EDITH 3-ethoxy-1,2,4-dithiazoline-5-one

EDTA ethylenediaminetetraacetic acid

EI electron impact (mass spectrometry)

ENU ethylnitrosourea

ESI electrospray ionization (mass

spectrometry)

Et ethyl

FAB fast atom bombardment (mass

spectrometry)

FAM 5-carboxyfluorescein

FDA (United States) Food and Drug

Administration

FFT fast Fourier transform

FLB formamide loading buffer

Fm fluorenylmethyl

FMN flavin mononucleotide

FMOC 9-fluorenylmethoxycarbonyl

For formyl

FPLC fast protein liquid chromatography

Fpmp 1-(2-fluorophenyl)-

4-methoxypiperidin-4-yl

FRET fluorescence resonance energy

transfer

FTICR Fourier-transform ion-cyclotron resonance (mass spectrometry)

G guanine; guanosine

GDP guanosine 5'-diphosphate

GMP guanosine $5^{\prime}$-monophosphate

GTP guanosine 5 '-triphosphate

HATU $O$-(7-azabenzotriazol-1-yl)-1,1,3,3tetramethyluronium hexafluorophosphate

HBTU 2-(1H-benzotriazol-1-yl)-1,1,3,3-

tetramethyluronium hexafluorophosphate

HEC hydroxyethylcellulose

HEG hexa(ethylene glycol)

HEPES $N$-(2-hydroxyethyl)piperazine- $N N^{\prime}$ (2-ethanesulfonic acid)

HEPPSO $N$-(2-hydroxyethyl)piperazine$N^{\prime}$-(2-hydroxypropanesulfonic acid) 
HMDS hexamethyldisilazane

HMFS $N$-[9-(hydroxymethyl)-2-

fluorenyl]succinamic acid

HMQC heteronuclear multiple quantum coherence

HNA hexitol nucleic acid

HOAt 7-aza-1-hydroxybenzotriazole

HOBt or HOBT 1-hydroxybenzotriazole

3-HPA 3-hydroxypicolinic acid

HPLC high-performance liquid

chromatography

HPMC hydroxypropylmethylcellulose

HQDA hydroquinone- $O, O^{\prime}$-diacetic acid

HRMS high-resolution mass spectrometry

HSDIS hydroxystyryldiisopropylsilyl

HSDMS hydroxystyryldimethylsilyl

HSQC heteronuclear single quantum coherence

HZ hydrazine

I inosine

$\boldsymbol{i}$-Bu isobutyl; isobutyryl

ICS isocarbostyril

IEC ion-exchange chromatography

IMT imidazolium triflate

$\boldsymbol{i}$-Pr isopropyl

IR infrared

IRAA internal reference amino acid

ITC isothermal titration calorimetry

IUB International Union of Biochemistry

IUPAC International Union of Pure and Applied Chemistry

JOE 6-carboxy-4', $5^{\prime}$-dichloro-2', $7^{\prime}$ dimethoxyfluorescein

LCAA long-chain alkylamine

LNA locked nucleic acid

LSIMS liquid secondary-ion mass spectrometry

L-TOF linear time-of-flight (mass spectrometry)

MALDI matrix-assisted laser desorption/ionization (mass spectrometry)

MAST [bis(2-methoxyethyl)amino]sulfur trifluoride

MB minor groove binder

MB-ODN minor groove binderoligodeoxyribonucleotide conjugate

MBHA $p$-methylbenzhydrylamine

MC Monte Carlo

mCPBA meta-chloroperoxybenzoic acid (also 3-chloroperoxybenzoic acid)

MD molecular dynamics

MDMP 1,5-dimethoxylcarbonyl-3-

methoxypentan-3-yl

Me methyl
MeNPOC methylnitropiperonyloxy-

carbonyl

Me-P methylphosphonate

MES 2-( $N$-morpholino)ethanesulfonic acid

MICS methylisocarbostyril

MM molecular mechanical

MMI methylenemethylimino

MMTr 4-monomethoxytrityl

MOPS 3-( $N$-morpholino)propane sulfonic

acid

MOTr 4-methoxy-4'-octyloxytrityl

MOX 9-(p-anisyl)xanthen-9-yl

MPC $N$-methylpyrrolecarboxamide

MPLC medium-pressure liquid

chromatography

mRNA messenger ribonucleic acid

MS mass spectrometry

MSNT 1-(mesitylene-2-sulfonyl)-3-nitro-

1,2,4-triazole

MTHP 4-methoxytetrahydropyran-4-yl

MTMB 2-(methylthiomethoxymethyl)

butyryl

MTMT 2-(methylthiomethoxymethyl)

benzoyl

MTPI methyltriphenoxyphosphonium

iodide

MWCO molecular weight cutoff

NAB nucleic acid builder (computer program)

NAIM nucleotide analog interference mapping

NAIS nucleotide analog interference suppression

NBOM 2-nitrobenzyloxymethyl

NBT 5-nitrobenzimidazolium triflate;

nitroblue tetrazolium

n-Bu $n$-butyl

NDB Nucleic Acid Database

NDP nucleoside diphosphate

NDPK nucleoside diphosphate kinase

NHS $N$-hydroxysuccinimide

NMI $N$-methylimidazole

NMM $N$-methylmesoporphyrin

NMP nucleoside monophosphate;

$\mathrm{N}$-methylpyrrolidinone

NMR nuclear magnetic resonance

NOE nuclear Overhauser effect

NOESY nuclear Overhauser effect

spectroscopy

N-P phosphoramidate

NP1 nuclease P1

NPE 2-(4-nitrophenyl)ethyl

NPEOC 2-(4-nitrophenyl)ethoxycarbonyl

NPOC nitrophenyloxycarbonyl 
NPPOC 2-(2-nitrophenyl)

propoxycarbonyl

NRC Nuclear Regulatory Commission

nt nucleotide

NTP nucleoside triphosphate

ODN oligodeoxyribonucleotide

PA picolinic acid

Pac or pac phenoxyacetyl

PACE phosphonoacetate

PAGE polyacrylamide gel electrophoresis

PAL peptide amide linker (resin)

PAM 4-hydroxymethylphenylamido-

methyl (resin)

PAPOC $p$-phenylazophenyloxycarbonyl

PAPS 3'-phosphoadenosine-

5'-phosphosulfate

PASS product-anchored sequential

synthesis

PBS phosphate-buffered saline

PCR polymerase chain reaction

PDITC phenylene diisothiocyanate

PEG polyethylene glycol

PEG-MME polyethylene glycol-

monomethyl ether

PEO polyethylene oxide

PEP phosphoenolpyruvate

PFP pentafluorophenyl

PFPC bis(pentafluorophenyl) carbonate

Ph phenyl

PICS 7-propynyloxycarbostyril

PIM 7-propynyl-3-methylisocarbostyril

PIPES piperazine- $N, N^{\prime}-$

bis(2-ethanesulfonic acid)

PMSF phenylmethylsulfonyl fluoride

PMT photomultiplier tube

pn or PN phosphoramidate (linkage)

PNA peptide nucleic acid

PNK polynucleotide kinase

pnODN oligodeoxyribonucleotide

$\mathrm{N}^{\prime} \rightarrow \mathrm{P}^{\prime}$ phosphoramidate

PNP purine nucleoside phosphorylase

po or PO phosphodiester (linkage)

Pr propyl

PPA or PPG pyrazolo[3,4- $d]$ pyrimidine

analog of $\mathrm{dA}$ or $\mathrm{dG}$

ps or PS phosphorothioate (linkage)

PS polystyrene

PTFE polytetrafluoroethylene

PTMT 2-(isopropylthiomethoxymethyl)

benzoyl

Pu purine

Pv pivaloyl

Px 9-phenylxanthen-9-yl (pixyl)

PX paranemic cross-over

Py pyrimidine
PyAOP 7-aza-benzotriazol-1-yl-

oxytripyrrolidinophosphonium

hexafluorophosphate

PyBOP $1 H$-benzotriazol-1-yl-

oxytripyrrolidinophosphonium

hexafluorophosphate

QM quantum mechanics or quantum

mechanical

QNAIM quantitative nucleotide analog

interference mapping

$\mathbf{R}, \mathbf{R}^{\prime} \ldots \quad$ alkyl groups

$\boldsymbol{R}_{\mathbf{f}} \quad$ retention factor (TLC)

RFP radiofrequency plasma

RFLP restriction fragment length

polymorphism

rMD restrained molecular dynamics

RMSD root mean squared deviation

RNA ribonucleic acid

RNAi RNA interference

RNAP RNA polymerase

RNase ribonuclease

RNP ribonucleoprotein

ROX 5-(and 6-)carboxy-X-rhodamine

RP-HPLC reversed-phase

high-performance liquid chromatography

RT-PCR reverse transcription polymerase

chain reaction

SA sinapinic acid

SDS sodium dodecyl sulfate

sec-Bu sec-butyl

SELEX Systematic Evolution of Ligands

by Exponential Enrichment

SET 5-ethylthio- $1 H$-tetrazole

siRNA small interfering RNA

SNP single nucleotide polymorphism

S-PACE thiophosphonoacetate

3SR self-sustained sequence replication

SSC sodium chloride/sodium citrate

(buffer)

SSCP single-stranded conformational

polymorphism

ssDNA single-stranded DNA

SSPE saline sodium phosphate/EDTA

(buffer)

STM scanning tunneling microscopy

STR short tandem repeats

SVPD or SVP snake venom

phosphodiesterase

T thymidine; thymine

T-jump temperature-jump (relaxation)

tac tert-butylphenoxyacetyl (also $t$-PAC)

TAE Tris/acetate/EDTA (buffer)

TAMRA 5-(and 6-)carboxy- $N, N, N^{\prime}, N^{\prime}-$

tetramethylrhodamine

TBAF tetrabutylammonium fluoride 
TBDMS tert-butyldimethylsilyl

TBE Tris/borate/EDTA (buffer)

TBF 4-[17-tetrabenzo $(a, c, g, i)$

fluorenylmethyl]

TBS Tris-buffered saline

$\boldsymbol{t}$-Bu tert-butyl

TBAF tetrabutylammonium fluoride

TBAP tetrabutylammonium phosphate (also pyrophosphate or dihydrogenphosphate)

TBE Tris/borate/EDTA (buffer)

TCA trichloroacetic acid

TCEP tris-(2-carboxyethyl)phosphine

TDP thymidine $5^{\prime}$-diphosphate

TE Tris/EDTA (buffer)

TEA triethylamine (also $\left.\mathrm{Et}_{3} \mathrm{~N}\right)$

TEAA triethylammonium acetate

TEAB triethylammonium bicarbonate

TEMED $N, N, N^{\prime}, N^{\prime}$-tetramethyl-

ethylenediamine

TES $N$-tris(hydroxymethyl)methyl-2aminoethanesulfonic acid

TFA trifluoroacetic acid

TFC triplex-forming circle

TFMSA trifluoromethanesulfonic acid

TFO triplex-forming oligonucleotide

TFP tetrafluorophenyl

THAP $2^{\prime}, 4^{\prime}, 6^{\prime}$-trihydroxyacetophenone

THF tetrahydrofuran

THP tetrahydropyran-2-yl

TIPS triisopropylsilyl;

tetraisopropyldisiloxane

TLC thin-layer chromatography

TMP 3,4,7,8-tetramethyl-1,10-

phenanthroline; thymidine

5 '-monophosphate;

trisodium trimetaphosphate
TMS trimethylsilyl

TMSE trimethylsilylethyl

TMS-OTf trimethylsilyl

trifluoromethanesulfonate

TMTr trimethoxytrityl

TNT terminal deoxyribonucleotide

transferase

TOCSY total correlation spectroscopy

TOF time-of-flight (mass spectrometry)

Tol toluoyl

TOM [(triisopropylsilyl)oxy]methyl

$\boldsymbol{t}$-PAC tert-butylphenoxyacetyl (also tac)

TPP triphenylphosphine

Tr diphenylmethyl (trityl)

tRNA transfer ribonucleic acid

TREAT-HF triethylammonium

trihydrofluoride

Tris tris(hydroxymethyl)aminomethane

TROSY transverse relaxation-optimized spectroscopy

Ts $\quad p$-toluenesulfonyl (tosyl)

TsOH toluene-4-sulfonic acid

TTP thymidine 5 '-triphosphate

TX triple cross-over

U uracil; uridine

UDP uridine $5^{\prime}$-diphosphate

UMP uridine 5'-monophosphate

UTP uridine 5'-triphosphate

UV ultraviolet

VNTR variable number tandem repeats

XAL xanthen alkonic acid (linker/resin)

YDBR yeast debranching enzyme
Abbreviations and Useful Data

A.1.5 